\title{
Numerical simulation of Brownian particles in optical force fields
}

Giorgio Volpe Giovanni Volpe 


\title{
Numerical Simulation of Brownian Particles in Optical Force Fields Giorgio Volpe ${ }^{\mathrm{a}}$, Giovanni Volpe ${ }^{* b}$ \\ ${ }^{a}$ Institut Langevin, ESPCI ParisTech, CNRS UMR7587, 1 rue Jussieu, 75005 Paris, France; \\ ${ }^{\mathrm{b}}$ Physics Department, Bilkent University, Cankaya, 06800 Ankara, Turkey
}

\begin{abstract}
Optical forces can affect the motion of a Brownian particle. For example, optical tweezers use optical forces to trap a particle at a desirable position. Using more complex force fields it is possible to generate more complex configurations. For example, by using two optical traps placed next to each other, it is possible to obtain a bistable potential where a particle can jump between the two potentials with a characteristic time scale. In this proceeding, we discuss a simple finite difference algorithm that can be used to simulate the motion of a Brownian particle in a one-dimensional field of optical forces.
\end{abstract}

Keywords: optical forces, Brownian motion, stochastic differential equations, numerical simulations, Kramers transitions

\section{INTRODUCTION}

We present a simple algorithm to simulate a Brownian particle in a one dimensional field of optical forces, as discussed in more detail in Ref. [1]. We provide an implementation of this algorithm using MatLab ${ }^{\circledR}$, because this language is widely employed in the science and engineering. All algorithms can also be translated straightforwardly in the freeware programming languages SciLab [2] or Octave.

In the first place, it is useful to simulate the motion of a Brownian particle in a field of optical forces to gain insight into how an optical trap works; since optical traps have found a widespread set of applications in fields as diverse as, e.g., cooling of single atoms, colloidal physics and biophysics, it can be useful to understand how these optical traps work in simple and inexpensive simulations. In second place, an optically trapped particle constitutes a simple example of a stochastic phenomenon whose evolution is determined by both stochastic (the Brownian motion) and deterministic (the optical forces) forcing terms and can, therefore, be used as a model system for more general stochastic phenomena.

\section{THEORETICAL MODEL}

A Brownian particle in a generic field of force can be described by the Langevin equation in one dimension $[1,3]$ :

$$
m \frac{d^{2}}{d t^{2}} x(t)=-\gamma \frac{d}{d t} x(t)+F(x(t), t)+\sqrt{2 k_{B} T \gamma} W(t)
$$

where $x(t)$ is the trajectory of the particle, $m$ is the particle mass, $\gamma$ is the friction exerted by the surrounding medium on the particle, $F(x(t), t)$ is an optical force acting on the particle that can vary both in space and time, $k_{B} T$ is the thermal energy unit, $k_{B}$ is the Boltzmann constant, $T$ is the absolute temperature and $W(t)$ is a Wiener process.

For microscopic particles immersed in a liquid, viscous forces are several orders of magnitude larger than inertial forces, i.e., the particle is in a low-Reynolds-number regime [4]. The Reynolds number is the ratio between inertial and viscous forces acting on an object moving in a fluid. If the object has a characteristic dimension $L$ and is moving at velocity $v$ through a fluid with viscosity $\eta$ and density $\rho$, its Reynolds number is $\mathrm{Re}=L v \rho / \eta$. In the low-Reynolds-number regime, i.e for $\operatorname{Re}<1$, the viscosity dominates over inertia. Considering, for example, an $E$. coli bacterium swimming in water

[5], $L \approx 1 \mu \mathrm{m}, v \approx 30 \mu \mathrm{m} / \mathrm{s}, \eta=0.001$ Pas and $\rho=1000 \mathrm{~kg} / \mathrm{m} 3$, so that $\operatorname{Re}=3 \mathrm{e}-5 \ll 1$. One of the most striking aspects of low Reynolds number phenomena is that the speed of an object is solely determined by the forces acting on it at the moment; a good introduction to how is life at low Reynolds numbers can be found in Ref. [4]. In general, most optical

"'giovanni.volpe@fen.bilkent.edu.tr; http://www.softmatter.bilkent.edu.tr

Optical Trapping and Optical Micromanipulation X, edited by Kishan Dholakia, Gabriel C. Spalding, Proc. of SPIE Vol. 8810, 88102R - (c) 2013 SPIE · CCC code: 0277-786X/13/\$18 - doi: 10.1117/12.2022517 
manipulation experiments take place at low Reynolds numbers, the exceptions being experiments in fluids with very low viscosity such as air [6]. Therefore, in Eq. (1) we can drop the inertial term obtaining:

$$
\frac{d}{d t} x(t)=\frac{1}{\gamma} F(x(t), t)+\sqrt{2 D} W(t)
$$

where $D$ is the Stokes-Einstein diffusion coefficient.

Eq. (2) is one of the simplest examples of a stochastic differential equation $[7,8]$. In general, stochastic differential equations are obtained from ordinary differential equations adding a noise term, i.e. the Wiener process $W(t)$. $W(t)$ is characterized by the following properties:

1. the mean $\langle W(t)>=0$ for all $t$;

2. $<W(t)^{2}>=1$ for each value $t$;

3. $W\left(t_{l}\right)$ and $W\left(t_{2}\right)$ are independent of each other for $t_{1}$ different from $t_{2}$.

Because of these properties, a white noise is not a standard function. In particular, $W(t)$ is almost everywhere discontinuous and has infinite variation. In an intuitive picture, it can be seen as the continuous-time equivalent of a discrete sequence of independent random numbers.

\section{SIMULATION OF WHITE NOISE}

In order to simulate Eq. (2) it is possible to employ a finite difference algorithm, where the continuous-time solution $x(t)$ is approximated by a discrete time sequence $x_{i}$ evaluated at times $t_{i}=i \Delta t$. This is in practice done by doing the following substitutions in Eq. (2) [1,7]:
1. $x(t)$

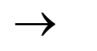
$x_{i}$
2. $\frac{d}{d t} x(t)$
$\rightarrow$
$\frac{x_{i}-x_{i-1}}{\Delta t}$
3. $W(t)$

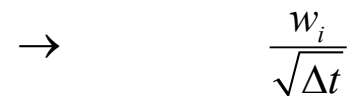

where $w_{i}$ is a sequence of Gaussian random numbers with the following properties:

1. the mean $<w_{i}>=0$;

2. $\left\langle w_{i}^{2}>=1\right.$;

3. $<w_{i} w_{j}>=0$ for $i$ different from $j$.

Notice how these properties mimic the ones of the Wiener process given above.

By making the aforementioned substitutions in Eq. (2) one obtains the following finite difference equation:

$$
x_{i}=x_{i-1}+\frac{1}{\gamma} F\left(x_{i}, i\right) \Delta t+\sqrt{2 D \Delta t} w_{i}
$$

which can now be solved numerically.

\section{1D SIMULATION CODE}

In this section we present the MatLab ${ }^{\circledR}$ code that implements Eq. (3) following the codes provided with Ref. [1]. In particular we will study the case of a mono- and bi-stable optical trap. 


\subsection{Monostable optical trap}

This function implements Eq. (3) in one dimension for the case of a monostable optical trap $F\left(x_{i}, i\right)=-k x_{i}$, where $k$ is the trap stiffness. Inputs: number of samples $\mathrm{N}$, timestep $\mathrm{Dt}$, initial position $\mathrm{x} 1$, particle radius $\mathrm{R}$, temperature $\mathrm{T}$, fluid viscosity eta, and trap stiffness $\mathrm{k}$. Outputs: particle position $\mathrm{x}$ in meters and time $t$ in seconds. Figure 1 shows the result of this simulation.
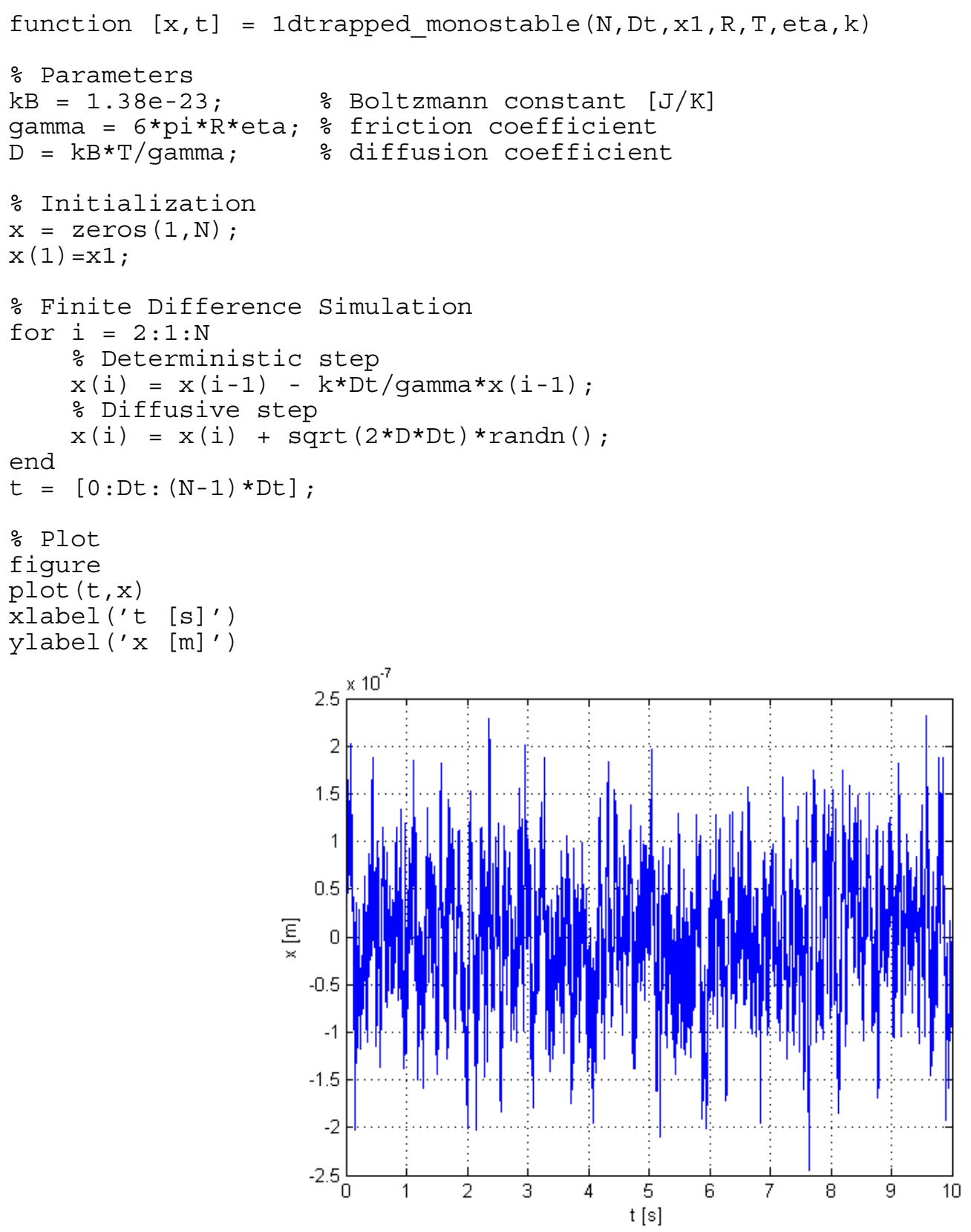

Figure 1. Simulation of an optically trapped particle in one dimension with the following parameters: $N=1 e+4 ; D t=$ $1 \mathrm{e}-3[\mathrm{~s}] ; \mathrm{x} 1=0[\mathrm{~m}] ; \mathrm{R}=1 \mathrm{e}-6[\mathrm{~m}] ; \mathrm{T}=300[\mathrm{~K}] ;$ eta $=0.001[\mathrm{~Pa} \mathrm{~s}-$ water $] ; \mathrm{kx}=$ $1 e-6[\mathrm{~N} / \mathrm{m}]$. 


\subsection{Bistable optical trap: Kramers transitions}

This function implements Eq. (3) in one dimension for the case of a bistable optical trap $F\left(x_{i}, i\right)=-a x_{i}^{3}+b x_{i}$, where $a$ and $b$ are two parameters which define the potential of the double well [29]. Inputs: number of samples $\mathrm{N}$, timestep $\mathrm{Dt}$, initial position $\mathrm{x} 1$, particle radius $\mathrm{R}$, temperature $\mathrm{T}$, fluid viscosity et $\mathrm{a}$, and double well parameters $\mathrm{a}$ and $\mathrm{b}$. Outputs: particle position $\mathrm{x}$ in meters and time $t$ in seconds. Figure 2 shows the result of this simulation.
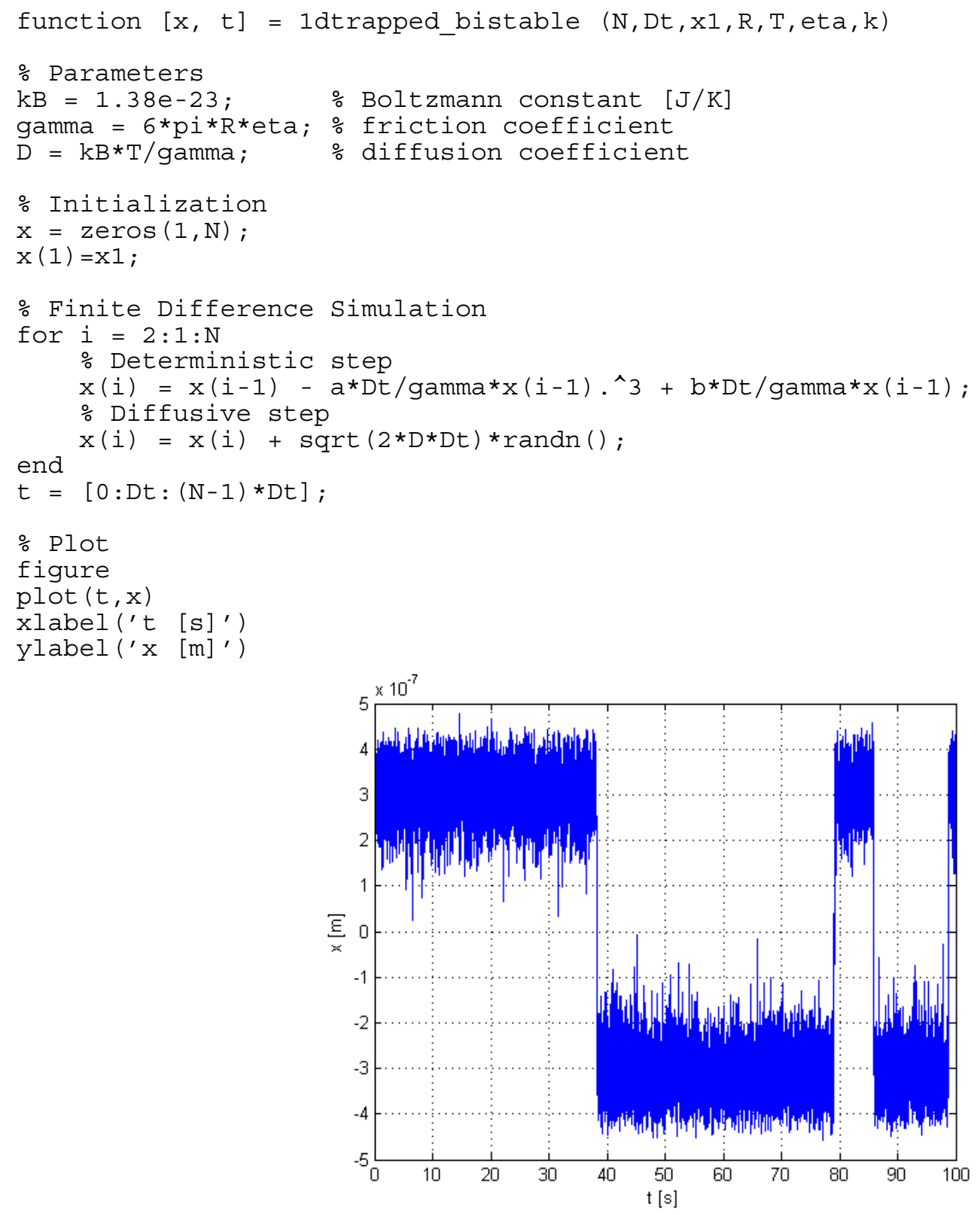

Figure 1. Simulation of a Brownian particle trapped in a bistable potential in one dimension with the following parameters: $\mathrm{N}=2 \mathrm{e}+6 ; \mathrm{Dt}=1 \mathrm{e}-3[\mathrm{~s}] ; \mathrm{x} 1=0[\mathrm{~m}] ; \mathrm{R}=1 \mathrm{e}-6[\mathrm{~m}] ; \mathrm{T}=300[\mathrm{~K}] ;$ eta $=0.001[\mathrm{~Pa} \mathrm{~s}-$ water $] ; \mathrm{a}=1 \mathrm{e} 7\left[\mathrm{~N} / \mathrm{m}^{\wedge} 3\right] ; \mathrm{b}=1 \mathrm{e}-6[\mathrm{~N} / \mathrm{m}]$. 


\section{DIFFUSION GRADIENTS}

In the presence of diffusion gradients, which arise naturally, e.g., when a Brownian particle is trapped near another particle or near a surface, some additional correction are needed in order to account for a spurious drift $[9,10,11,12]$. This spurious drift results from the fact that the Wiener process is multiplied by a function of the particle position and is needed to correct an indetermination inherently occurring in stochastic differential equations with multiplicative noise. How to deal with these cases is explained in Refs. [13,14]. Similar considerations hold also in the case of diffusion gradients induced by, e.g., chemical gradients or temperature gradients $[15,16,17]$.

\section{FURTHER NUMERICAL EXPERIMENTS}

Using the code provided in Section 4, it is possible to simulate an optically trapped particle under various conditions and verify, e.g., that the particle variance in the optical trap is inversely proportional to the trap stiffness [18]. Furthermore, it can be interesting to apply these simulation techniques to simulate and study the behavior of a Brownian particle in more complex force fields: for example, a Brownian particle in the presence of external forces or torques $[19,20,21,22,23,24,25,26,27,28]$. Also time-varying force fields can be considered, which leads to more complex phenomena, such as stochastic resonant damping [30] and stochastic resonance [31].

\section{REFERENCES}

[1] Volpe, G. and Volpe, G., "Simulation of a Brownian particle in an optical trap," Am. J. Phys. 81, 224-230 (2013).

[2] Scilab $<$ www.scilab.org/>.

[3] Nelson E., Dynamical Theories of Brownian Motion (Princeton University Press, Princeton, NJ, 1967).

[4] Purcell, E. M., "Life at low Reynolds numbers," Am. J. Phys. 45, 3-11 (1977).

[5] Berg, H., E. coli in Motion (Springer Verlag, New York, NY, 2004).

[6] Li, T., Kheifets, S., Medellin, D., Mark G. Raizen, M. G., "Measurement of the instantaneous velocity of a brownian particle," Science 328, 1673-1675 (2010).

[7] Øksendal, B., Stochastic Differential Equations, 6th ed. (Springer, Heidel- berg, 2003).

[8] Kloeden, P. E., and Platen, E., Numerical Solution of Stochastic Differential Equations (Springer, Heidelberg, 1999).

[9] Ermak, D. L., and McCammon, J. A., "Brownian dynamics with hydrodynamic interactions," J. Chem. Phys. 69, 1352-1361 (1978).

[10] Lançon, P., Batrouni, G., Lobry, L., and Ostrowsky, N., "Drift without flux: Brownian walker with a spacedependent diffusion coefficient," Europhys. Lett. 54, 28 (2001).

[11]Lau, A. W. C., and Lubensky, T. C., "State-dependent diffusion: Thermodynamic consistency and its path integral formulation," Phys. Rev. E 76, 011123 (2007).

[12] Hottovy, S., Volpe, G., and Wehr, J., "Noise-Induced Drift in Stochastic Differential Equations with Arbitrary Friction and Diffusion in the Smoluchowski-Kramers Limit," J. Stat. Phys. 146, $762-773$ (2012).

[13] Volpe, G., Helden, L., Brettschneider, T., Wehr, J., and Bechinger, C., "Influence of Noise on Force Measurements," Phys. Rev. Lett. 104, 170602 (2010).

[14] Brettschneider, T., Volpe, G., Helden, L., Wehr, J., and Bechinger, C., "Force measurement in the presence of Brownian noise: Equilibrium-distribution method versus drift method," Phys. Rev. E 83, 041113 (2011).

[15] Hottovy, T., Volpe, G., and Wehr, J., "Thermophoresis of Brownian particles driven by coloured noise," EPL 99, $60002(2012)$.

[16] Ebbens, S. J., and Howse, J. R., "In pursuit of propulsion at the nanoscale," Soft Matter 6, 726-738 (2010).

[17] Howse, J. R., Jones, R. A. L., Ryan, A. J., Gough, T., Vafabakhsh, R., and Golestanian, R., "Self-motile colloidal particles: from directed propulsion to random walk," Phys. Rev. Lett. 99, 048102 (2007).

[18] Volpe, G., Wehr, J., Rubi, J. M., and Petrov, D., “Thermal noise suppression: how much does it cost?” J. Phys. A: Math. Theor. 42, 095005 (2009).

[19] Ashkin, A., "Optical trapping and manipulation of neutral particles using lasers," Proc. Natl. Acad. Sci. U.S.A. 94, 4853-4860 (1997). 
[20] Berg-Sorensen, K., and Flyvbjerg, H., "Power spectrum analysis for optical tweezers," Rev. Sci. Instrum. 75, 594-612 (2004).

[21] Rohrbach, A., Tischer, C., Neumayer, D., Florin, E.-L., and Stelzer, E. H. K., "Trapping and tracking a local probe with a photonic force microscope,” Rev. Sci. Instrum. 75, 2197-2210 (2004).

[22] Bishop, A. I., Nieminen, T. A., Heckenberg, N. R., and Rubinsztein-Dunlop, H., "Optical application and measurement of torque on microparticles of isotropic nonabsorbing material," Phys. Rev. A 68, 033802 (2003).

[23] La Porta, A., and Wang, M. D., "Optical torque wrench: Angular trapping, rotation, and torque detection of quartz microparticles,” Phys. Rev. Lett. 92, 190801 (2004).

[24] Volpe, G., and Petrov, D., "Torque detection using brownian fluctuations," Phys. Rev. Lett. 97, 210603 (2006).

[25] Volpe, G., Volpe, G., and Petrov, D., "Brownian motion in a nonhomogeneous force field and photonic force microscope," Phys. Rev. E 76, 061118 (2007).

[26] Volpe, G., Volpe, G., and Petrov, D., "Singular-point characterization in microscopic flows," Phys. Rev. E 77, 037301 (2008).

[27] Borghese, F., Denti, P., Saija, R., Iatì, M. A., and Maragó, O. M., "Radiation torque and force on optically trapped linear nanostructures,” Phys. Rev. Lett. 100, 163903 (2008).

[28] Pesce, G., Volpe, G., De Luca, A. C., Rusciano, G., and Volpe, G., "Quantitative assessment of nonconservative radiation forces in an optical trap," EPL 86, 38002 (2009).

[29] Gammaitoni, L., Hänggi, P., Jung, P., and Marchesoni, F., "Stochastic resonance," Rev. Mod. Phys. 70, 223287 (1998).

[30] McCann, L. I., Dykman, M., and Golding, B., "Thermally activated transitions in a bistable three-dimensional optical trap," Nature 402, 785-787 (1999).

[31] Volpe, G., Perrone, S., Rubi, J. M., and Petrov, D., "Stochastic resonant damping in a noisy monostable system: Theory and experiment," Phys. Rev. E 77, 051107 (2008). 